\title{
Correction to: Brentano's The Teaching of Jesus 100 Years Later: An Historical Introduction
}

\section{Correction to:}

Chapter 1 in: F. Brentano, The Teaching of Jesus and its

Enduring Significance, Primary Sources in Phenomenology, https://doi.org/10.1007/978-3-030-68912-4_1

This chapter was inadvertently published with the Dr. Brentano as the chapter author instead of Dr. Schaefer. It is now corrected to reflect Dr. Schaefer as the author. 